\title{
DÉNOTATION ET CONNOTATION DANS LA LANGUE DES SCHOLIES *
}

PAscale Hummel

Los escolios añaden al texto principal un segundo, o secundario, texto, gramatical o filológico. A veces añaden también un contenido que puede ser considerado superfluo, irrelevante o incluso innecesario por los filólogos modernos. Los comentaristas antiguos, de hecho, elegían qué querían comentar y cómo querían hacerlo. En la mayor parte de los casos, consideraron los textos más antiguos desde el punto de vista de su propio tiempo. Los escolios, ciertamente, no reflejan una objetividad expositiva que se limitaría a explicar el significado verdadero y, en la medida de lo posible, único. Los gramáticos comentan aquello que sus mentalidades o sus lenguajes les llevan a comentar. El resultado filológico, por consiguiente, parece una mixtura de objetividad y subjetividad. Cuando un gramático griego de la edad alejandrina comenta un texto griego de una edad anterior, ¿de qué manera se pone la lengua griega bajo un enfoque diferente? El lenguaje técnico de los escoliastas ¿aporta a los textos comentados más "connotación” o más "exposición”? ¿Cómo se funden el plano sincrónico (el del comentario) y el diacrónico (el del texto comentado), y qué clase de significado es entonces expresado o presentado?

Palabras clave: escolios; connotación / denotación; comentarios griegos; objetividad / subjetividad; diacronía / sincronía; exégesis; metalenguajes; papeleteo; filología
The scholia add a second or secondary (grammatical; philological) text to the main (literary) text. They sometimes also add a meaning which might be considered (by modern philologists) as superfluous, irrelevant, or even unnecessary. The ancient commentators actually choose what they want to comment upon and how they want to do so. They mostly see the anterior texts from the point of view of their own synchrony and understanding. Scholia certainly don't reflect any denotative objectivity, which would just unfold the true and unique (if possible) meaning. The grammarians comment what their minds or their languages lead them to comment upon. Therefore the philological result looks like a mixture of objectivity and subjectivity. When a Greek grammarian of the Alexandrian era comments upon a Greek text of a previous time, in which ways is the Greek language put into a changing perspective? Does the technical language of the scholiasts provide the commented texts with more connotation or more denotation? How do the synchrony (of the commentary) and the diachrony (of the texts commented upon) meet, and what kind of meaning is then expressed or made available?

Keywords: scholia; connotation / denotation; Greek commentaries; objectivity / subjectivity; diachrony / synchrony; exegesis; metalanguage; schedography; dittology; philology

Les scholies sont issues des commentaires antiques (notamment des zetemata péripatéticiens et des hypomnemata alexandrins) dont elles constituent

* Texte de la communication donnée lors du colloque «Fachtexte und Fachsprachen in der Antike», qui s'est tenu à Berlin en mars 2004.

EMERITA. Revista de Lingüística y Filología Clásica (EM) — ISSN 0013-6662 
des extraits. Elles se présentent sous la forme de lemmes. S'ajoutant aux textes qu'elles ont pour but d'expliquer, elles glosent un ou plusieurs des éléments les constituant. Leur visée est avant tout explicative, c'est-à-dire épexégétique : elles proposent le dépliage, autrement dit l'explicitation, du sens contenu dans des signifiants ou des constructions perçus a posteriori comme opaques. Elles sont au service des œuvres qu'elles prennent pour objet d'étude herméneutique ou grammatical. Le postulat initial est de la sorte dénotatif, le commentaire scholiographique ayant le statut de texte second dupliquant le texte premier sous la forme d'une longue paraphrase segmentée. Le dévoilement du sens «caché», dans la mesure où il ajoute du texte au texte, superpose, plus ou moins volontairement, un registre connotatif au registre dénotatif. Visant l'éclaircissement, la langue des commentateurs n'en véhicule pas moins des nuances inhérentes à leur propre synchronie. Le grec et le latin des exégètes n'est pas celui des textes, même grecs et latins, commentés. L'objectivité dénotative est par conséquent illusoire. Dans quelle mesure est-il possible d'apprécier le décalage linguistique entre la langue-commentaire et la langue commentée, notamment et surtout si elles représentent deux états chronologiques d'une même langue? Que commentent les commentateurs, sinon leur perplexité face à un texte que le temps a rendu obscur? À partir d'exemples empruntés au corpus scholiographique des deux langues et issus de tous les genres littéraires, il est possible d'apprécier la nature des obstacles linguistiques rencontrés par les exégètes et les moyens mis en œuvre pour les pallier ou les contourner. À la volonté de comprendre et d'expliquer qui structurellement oriente leur propos s'ajoute la spécificité du cadre, pour ainsi dire technique, qui conditionne (et restreint) l'usage de la langue. Sur quoi porte le commentaire : sur les obscurités dénotatives ou les lacunes connotatives? Et la langue des scholiastes elle-même, ajoute-t-elle de la dénotation ou de la connotation? Comment le sens sort-il indemne de l'intersection des impératifs de compréhension, d'interprétation, de mise en perspective diachronique et d'appropriation synchronique ; comment la langue s'accommode-t-elle de la rencontre problématique d'un usage respectivement littéraire (les textes) et technique (les scholies)?

Du point de vue de la chronologie, les scholies arrivent toujours après les textes classiques: pour les philologues modernes, cela va de soi; pour les philologues anciens, la question ne se pose pas tout à fait de la même manière. En quoi le facteur de la chronologie est-il pertinent et, plus fondamenta- 
lement encore, en quoi consiste-t-il exactement? Sauf dans la partie bio-historiographique de ce qu'on appelle les Vies, aucun commentateur ancien ne mentionne jamais le critère de la chronologie ou de la diachronie. Les textes de la tradition semblent reçus par les commentateurs presque sub specie aeternitatis, d'un point de vue en quelque sorte abstrait. Leur démarche, sans être à proprement parler achronique ou atemporelle, se situe en réalité à un tout autre plan que celui de la chronologie. Dès les premiers temps de la philologie, la discipline se donna des règles et des principes à elle-même. L'idée prédominante est celle d'une pérennité à la fois abstraite et absolue, valable avant tout à l'intérieur de sa propre sphère. La philologie est toujours et dans tous les cas métalinguistique: elle vient «après» et porte «sur» ce qui constitutivement la fonde. Les commentaires de la Renaissance, et même au delà, attestent, a posteriori et surtout a fortiori, que la philologie est pérenne d'une manière durable et répétitive. La philologie peut être dite dénotative au sens le plus général, dans la mesure où elle dénote l'héritage classique dans son extension la plus large; l'antiquité est son élément invariant et objectif de signification. La philologie peut être dite connotative, car elle ajoute au donné un élément d'appréciation secondaire; elle transforme le donné en commenté, le dénoté en connoté. Du point de vue de la philologie, le dénoté est toujours connoté; le noté est noté deux fois. La philologie prend acte du dénoté et simultanément le prend en charge. Elle donne réalité à l'idée organique d'une antiquité significative, en même temps qu'elle confère de l'étendue à une sorte de noyau sémiotique originel. Les textes grecs et latins sont proposés à la connaissance grammaticale comme un vaste réservoir de signes.

Les corpus des scholies inaugurent le commentaire perpétuel de l'antiquité entendue comme une longue chaîne de textes significatifs à l'infini. La philologie inaugure une relation de dépendance entre toutes les synchronies post-antiques et l'antiquité dont elles sont tributaires. Elle donne forme à l'idée de tradition. Son rôle premier, tel qu'elle le définit elle-même plus ou moins explicitement, est de transmettre, sans limite de durée ni de temps, le sens attaché pour ainsi dire au grand texte de l'antiquité. En copiant, répétant, commentant, glosant et expliquant à l'infini, la philologie fait durer les textes, leur donne une étendue et les met en perspective sans fin. Présentes dans les marges et entre les lignes des textes, les annotations des scholiastes apparaissent d'emblée comme une excroissance ou un accroissement. Elles augmentent le texte en même temps qu'elles le commentent. Elles l'encom-

EMERITA. Revista de Lingüística y Filología Clásica (EM) — LXXIII 1, 2005 ISSN 0013-6662 
brent même parfois, le surchargent et vont jusqu'à le déborder. Au sens littéral voire matériel du terme, les annotations sont, en quelque sorte par nature, une con-notation, c'est-à-dire une notation ajoutée.

Prises en bloc et comme un ensemble significatif en soi, les scholies sont inséparablement dénotatives et connotatives. Elles dénotent, c'est-à-dire désignent comme antérieurs les textes anciens qu'elles prennent pour objets; elles connotent, c'est-à-dire qu'elles ajoutent du sens par le commentaire. Elles prennent les textes comme objet primaire et les portent en quelque sorte à un état de réalité secondaire. Prises séparément, auteur par auteur ou œuvre par œuvre, les scholies livrent le fruit d'un long travail de sédimentation sur la tradition. Telles que les éditions modernes les présentent, elles proposent une lecture horizontale des couches successives de l'histoire exégétique. La verticalité des interlignes ou des marges est convertie en principe de juxtaposition généralisée. L'existence de différentes mains de commentateurs induit de plus une stratification linéaire requérant une lecture en quelque sorte à plusieurs niveaux. Dans tous les cas (tradition exégétique simple ou complexe) le texte-source continue d'exister indépendamment des excroissances parasites qu'il suscite. Comme le montrent ci-après les extraits des scholies à Homère ${ }^{1}$, le commentaire se situe d'emblée du côté de la prose non artistique, voire propose une sorte de traduction prosaïque de l'original poétique.

Les textes-sources, même lorsqu'il s'agit de prose, dans la mesure où ils sont du côté de la littérature, peuvent tous être dits poétiques. Et c'est précisément ce caractère poétique ou littéraire que le commentaire prend en charge pour le convertir ou l'expliquer. Dans le cas du lexique, le supplément connotatif qui préside au choix de tel terme plutôt que de tel autre constitue précisément l'occasion du commentaire, le scholiaste ramenant, voire réduisant, la connotation à la dénotation. Voici quelques exemples de termes composés:

B 450. $\pi \alpha 1 \varphi \alpha ́ \sigma \sigma o v \sigma \alpha] \pi \alpha ́ v \tau \eta \tau \grave{\alpha} \varphi \alpha ́ \eta ~ \alpha ُ i ́ \sigma \sigma o v \sigma \alpha$

$\varphi \tilde{\omega} \tau$

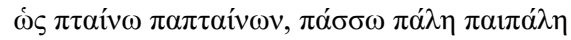

Dans cet exemple, le scholiaste décompose la forme composée en la ramenant à la stricte linéarité prosaïque; le propos est étymologiquement inté-

Scholia Graeca in Homeri Iliadem (scholia vetera), rec. H. Erbse, Berlin, W. de Gruyter, 1969-1988.

EMERITA. Revista de Lingüística y Filología Clásica (EM) — LXXIII 1, 2005 ISSN 0013-6662 
ressant, à défaut d'être tout à fait convaincant, et s'accompagne dans un second temps d'un développement sur les mots que l'auteur rapporte à la même famille. Le procédé de dépliage dénotatif du connotatif se retrouve dans les composés suivants:

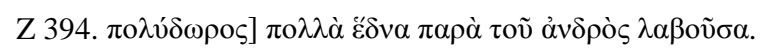

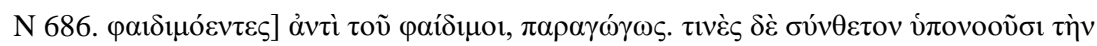

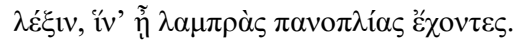

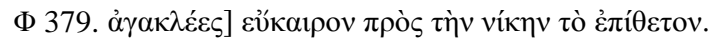

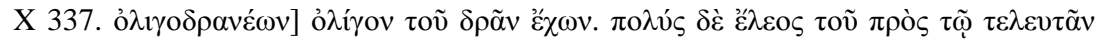

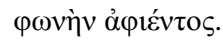

De même dans le corpus des scholies à Pindare ${ }^{2}$ :

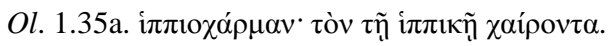

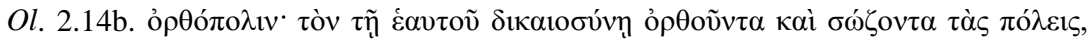

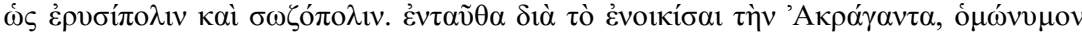

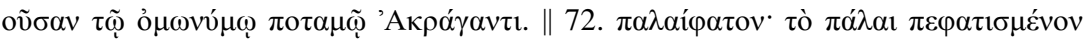

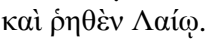

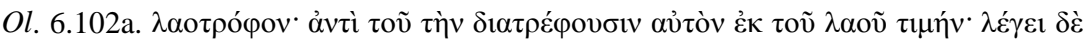

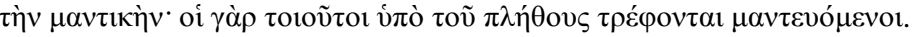

Dans le cas de ces composés, la connotation présente dans le texte-source sous la forme d'une hypostase lexicale est soumise par le commentateur à une sorte de conversion rétrospective ramenant la concision poétique à l'explicitation prosaïque. Le commentaire mérite bien en l'occurrence son nom d'exégèse, puisqu'il donne de l'étendue au texte de départ: il le déplie, le déploie et le convertit. Même dans le cas de la prose, les adjectifs composés font l'objet d'un soin particulier, comme dans les scholies au Contre Timarque d'Eschine par exemple:

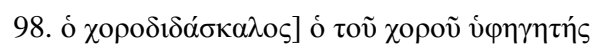

L'adjectif composé réunit presque idéalement toutes les caractéristiques de la connotation. À divers points de vue (morphosyntaxique, morpholexical, stylistique), le composé (adjectival, substantival ou verbal) est un condensé de sens, presque une icône ou un symbole de la poéticité. Il montre que bien écrire, c'est faire l'économie des mots, en user avec pertinence autant qu'avec modération. Les mêmes remarques valent pour les mots plus immédiatement appelés mots poétiques, à quelque catégorie morpholexicale qu'ils appartiennent. Dans les extraits suivants:

2 Scholia vetera in Pindari carmina, I, rec. A. B. Drachmann, Stuttgart \& Leipzig, B. G. Teubner, 1997.

EMERITA. Revista de Lingüística y Filología Clásica (EM) — LXXIII 1, 2005 ISSN 0013-6662 
pour Homère

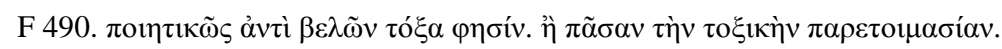

pour Euripide ${ }^{3}$, dans Les Phéniciennes

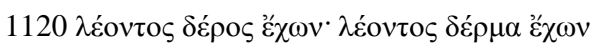

du même, dans Hippolyte

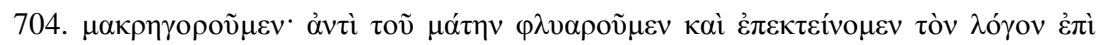

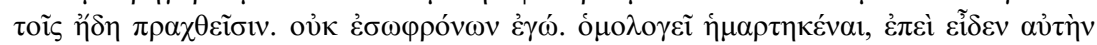

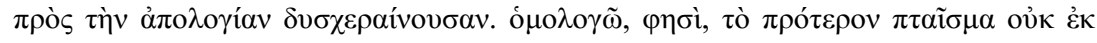

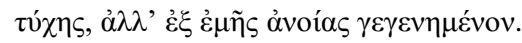

ou encore dans Oreste

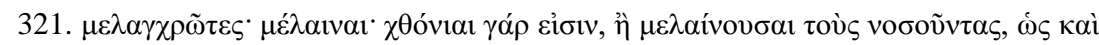

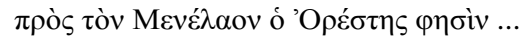

En elles-mêmes et pour ainsi dire prises génériquement, les scholies sont connotatives, car elles ajoutent du sens et du commentaire au texte premier. Par rapport au texte-source, elles réalisent une conversion dénotative du connotatif. Connotatives dans l'absolu donc, dénotatives d'un point de vue relatif.

Le transfert prosaïque se fait également pour des termes plus ordinaires, appartenant aux deux synchronies en présence, mais perçus par le commentateur comme affectés d'une nuance ne coïncidant pas avec l'usage de la synchronie de réception. Le décalage en la matière est moins entre poésie et prose qu'entre deux choix stylistiques. La remarque ne porte pas sur le signifiant et son excentricité, mais sur la simple nuance que le commentateur perçoit en plus ou en moins par rapport à l'usage de son temps. Le fait est assez patent dans les extraits suivants des scholies au Contre Timarque d'Eschine ${ }^{4}$ :

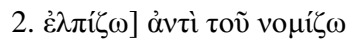

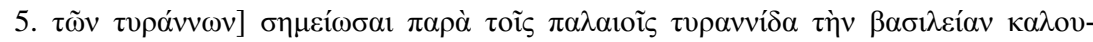

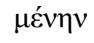

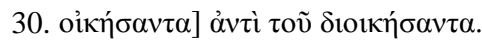

31. $\kappa \alpha \tau \alpha \gamma \varepsilon \lambda \alpha \dot{\sigma} \sigma \omega \varsigma] \dot{\alpha} \sigma \varepsilon \lambda \gamma \tilde{\omega} \varsigma$

51. $\pi \alpha \rho \rho \eta \sigma 1 \alpha \dot{\alpha} \zeta \varepsilon \tau \alpha 1] \sigma \alpha \varphi \tilde{\omega} \varsigma \lambda \varepsilon \dot{\gamma \varepsilon 1}$

3 Scholia Graeca in Euripidis tragoedias ex codicibus aucta et emendata, ed. G. Dindorf, Oxonii, E typographeo Academico, 1863.

4 Scholia Graeca in Aschinem et Isocratem ex codicibus aucta et emendata, ed. G. Dindorf, Hildesheim, H. A. Gerstenberg, 1970.

EMERITA. Revista de Lingüística y Filología Clásica (EM) — LXXIII 1, 2005 ISSN 0013-6662 


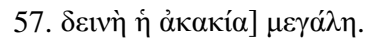

70. $\dot{v} \pi \varepsilon \rho \eta ́ \varphi \alpha v \alpha] \mu \varepsilon \gamma \alpha \dot{\lambda} \alpha$

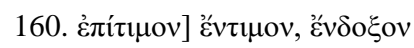

ou encore dans Isocrate

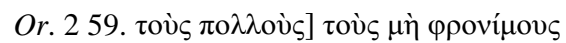

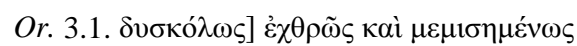

dans Apollonios de Rhodes ${ }^{5}$

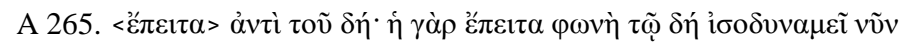

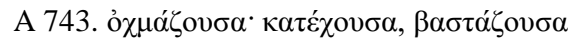

dans Euripide, Rhésus

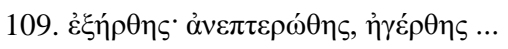

De même en latin, dans Juvénal ${ }^{6}$

Satire 2.11. Hispida: hirsuta $\|$ 46. (Defendit numerus): multitudo, id est: quia multi sunt, ideo non accusantur. ut Lucanus (V 260) || 124.1. (Segmenta) (Longos habitus): profusae uestes. 2. (Segmenta) (et longos): fimbriatas siue uittatas uestes, quibus matronae nou<a>e nuptae cooperiuntur. || 170. Sic praetextatos (referunt Artaxata mores): id est: mores, quos filii senatorum intra urbem habent, obsides in suis patriis redeuntes reportant, quos ceteri apud se ephebi discant.

Satire 6.203. Crudis: saturis, indigestis.

Satire 10.61. Iam strident ig(nes): id est: conflatur statua, ut pecunia inde fiat.

Dans ces exemples, la nuance entre le mot-source et la glose est si infime qu'elle pourrait presque échapper à un lecteur moderne. Le glissement se fait à l'intérieur de la sphère des synonymes, ou des équivalents usuels, les plus proches. La nécessité du commentaire est en l'occurrence moins épistémique que subjective; elle atteste, d'une certaine manière, le besoin d'explication ressenti par le commentateur lui-même. La scholie dans ce cas procède de la simple dittologie, c'est-à-dire de l'itération pour les besoins d'une traduction interne. L'impératif d'intelligibilité n'entre pas en compte ici. Comme l'explique C. Kerbrat-Orecchioni ${ }^{7}$ pour la langue française, la connotation d'une unité, ce n'est pas sa signification (ou compréhension) globale, mais l'ensemble des "composantes connotatives" d'un terme, à savoir certaines composantes seulement de sa signification, qui, loin d'être 1958.

5 Scholia in Apollonium Rhodium vetera, rec. C. Wendel, $2^{\mathrm{e}}$ éd., Berlin, Weidmann,

Scholia in Iuvenalem vetustiora, rec. P. Wessner, Leipzig, B. G. Teubner, 1931.

La connotation, Lyon, Presses universitaires de Lyon, 1977.

EMERITA. Revista de Lingüística y Filología Clásica (EM) — LXXIII 1, 2005 ISSN 0013-6662 
tenues pour les plus importantes, apparaissent comme des valeurs ajoutées, secondes, ou périphériques. Les constituants fondamentaux de la signification d'un terme sont les traits dénotatifs, ou sèmes, que dégage l'analyse en constituants minimaux. Le statut de connotation repose sur la nature particulière du signifié, à savoir l'appartenance à tel niveau de langue ou type de discours, la valeur affective, la valeur axiologique, l'image associée. Certaines valeurs sémantiques pour ainsi dire ajoutées apparaissent à la faveur de mécanismes associatifs (effets de la polysémie, des collocations, de l'allusion, etc.). En principe, la distinction est claire entre les traits de connotation stylistique et les traits dénotatifs, et la confusion possible entre connotation stylistique et trait axiologique. Connotation stylistique et connotation énonciative, qui informe sur l'énonciateur ${ }^{8}$, se trouvent étroitement liées, par l'intermédiaire de la connotation axiologique. Alors que la signification dénotative constitue le minimum nécessaire pour assurer la communication d'une notion, la signification connotative, proche de ce que certains linguistes appellent les associations extranotionnelles, peut être définie comme une sorte de halo associatif. L'association repose sur une affinité sémantique procédant de la paronymie, de l'analogie, de la synonymie, de la parasynonymie, en un mot d'affinités combinatoires. Selon les deux axes paradigmatique et syntagmatique, les relations de paronymie, synonymie, antonymie, hypéronymie, hyponymie, homonymie et polysémie peuvent être sources d'enrichissements connotatifs, que les deux signifiants correspondants soient co-présents dans l'énoncé (relation in praesentia), ou que l'un sollicite paradigmatiquement l'autre absent (association in absentia). Les significations implicites sont les valeurs connotées, et se répartissent en cinq classes: 1) effets phonétiques, rythmiques et syntaxiques, 2) connotations stylistiques, 3) connotations énonciatives, 4) valeurs associées, 5) valeurs implicites. Les connotations des classes 1,4 et 5 sont sémantiques, c'est-à-dire qu'elles fournissent des informations sur le référent de l'énoncé; les autres sont subjectives ou axiologiques.

Les scholies proposent une large palette de substitutions paradigmatiques et d'équivalences syntagmatiques. Le décalage entre la synchronie de départ et la synchronie d'arrivée pose également la question de deux états successifs d'une même langue, c'est-à-dire de deux états idiomatiques. Deux types

8 C. Kerbrat-Orecchioni, L'énonciation. De la subjectivité dans le langage, Paris, A. Colin, 1980.

EMERITA. Revista de Lingüística y Filología Clásica (EM) — LXXIII 1, 2005 ISSN 0013-6662 
de paramètres entrent en jeu ici: celui de l'idiotisme ${ }^{9}$ et celui de l'idiosyncrasie. L'idiotisme définit l'appartenance de chaque locuteur ou scripteur à une communauté linguistique donnée; l'idiosyncrasie est le coefficient d'engagement subjectif dans la langue. Les deux en l'occurrence sont des deux côtés, à savoir du côté du texte-source et du côté du commentaire ${ }^{10}$. Le poète ou le prosateur commenté relève dans l'absolu d'une synchronie idiomatique donnée; le scholiaste relève d'une autre synchronie. Et chacun, par l'acte même d'user de la langue à son gré, dessine sa propre idiosyncrasie, qui, sans être nécessairement un style, peut être défini plus simplement comme l'actualisation (au sens linguistique) de la langue par et à travers la parole, c'est-à-dire tout simplement sa réalisation effective, ajustée en l'occurrence au cadre particulier (à savoir respectivement littérature et exégèse) d'une application donnée. L'idée de style n'est jamais explicitement présente dans les scholies, sinon sous la forme du repérage des tropes, qui ressortit à la tradition rhétorique, où les figures sont comprises et employées comme un ornement connotant la beauté ${ }^{11}$. À partir du texte-source connotant (ou ornant) pour ainsi dire la dénotation, le commentaire se donne pour tâche de dénoter la connotation, comme dans les exemples suivants:

dans Homère

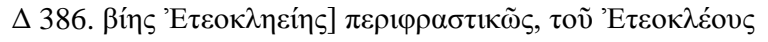

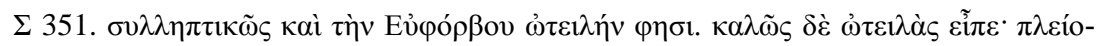

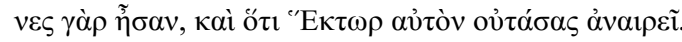

dans Euripide, Hippolyte

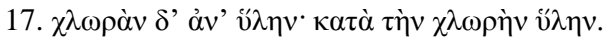

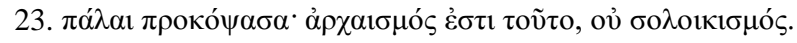

Les figures relevées dans ces passages sont la syllepse, la périphrase, l'antéposition, l'archaïsme ou la variation dialectale. Le scholiaste se contente de noter le phénomène, sans proposer d'autre explication que le cons-

9 G. Greciano, Signification et dénotation en allemand. La sémantique des expressions idiomatiques, Paris, Klincksieck, 1983.

10 Sur les caractéristiques stylistiques de certains érudits byzantins, voir Th. Hopfner, Thomas Magister, Demetrios Triklinios, Manuel Moschopulos. Eine Studie über ihren Sprachgebrauch in den Scholien zu Aischylos, Sophokles, Euripides, Aristophanes, Hesiod, Pindar und Theokrit, Wien, A. Hölder, 1912.

11 R. Griesinger, Die ästhetischen Anschauungen der alten Homererklärer dargestellt nach den Homerscholien, Tübingen, H. Laupp, 1907; R. Meijering, Literary and rhetorical theories in Greek scholia, Groningen, E. Forsten, 1987.

EMERITA. Revista de Lingüística y Filología Clásica (EM) — LXXIII 1, 2005 ISSN 0013-6662 
tat, pour lui évident, d'un choix stylistique ou rhétorique. Mêmes remarques et mêmes phénomènes dans les corpus des scholies latines:

\section{dans Virgile ${ }^{12}$}

Énéide I 1. ARMA multi uarie disserunt cur ab armis Vergilius coeperit, omnes tamen inania sentire manifestum est, cum eum constet aliunde sumpsisse principium, sicut in praemissa eius uita monstratum est. per 'arma' autem bellum significat, et est tropus metonymia. $\| 15$. FERTVR dicitur. et ingenti arte Vergilius, ne in rebus fabulosis aperte utatur poetarum licentia, quasi opinionem sequitur et per transitum poetico utitur more. \| 320. NVDA GENV nudum genu habens, ut si dicas ‘bonus animum'. et est Graeca figura, sed non ea quam diximus fieri per participium praeteriti temporis et casum accusatiuum; haec enim per nomen fit: quamuis ad unam significationem recurrant.

II 40. ANTE OMNES aut perissologia est, quia dixit 'primus'; aut 'ante omnes', id est uidentibus omnibus. || 111. EVNTES ire cupientes, ut cum canerem reges et proelia, id est cum canere uellem. et est figura Graeca, ubi statuisse aliquid pro inchoatione habetur.

dans Juvénal

Satire 1.88. Alea quando (hos animos): in ellipsin dicit hoc.

Satire 8.78. (Stratus humi palmes uiduas desiderat ulmos): iterum metaphoram a uitibus fecit, quae ex arboribus pendent nec se possunt sustinere sine arboribus, nisi fuerint adnexae.

Les figures relevées sont la métonymie, l'ellipse, la métaphore, la périssologie. Dans les scholies Bobiensia ${ }^{13}$ à Cicéron, le commentateur souligne l'intention rhétorique délibérée:

p. 81 ( $\$ 91$ ): O miserum, infelicem illum diem! — Oratorie deplorat etiam illud tempus quo damnatus est ambitus crimine, ut in eo desinat inuidiosus esse in quo miserandus existimetur.

p. 87, 1. 20: Qui omnia sacrificia nosset. — Ut incestum significaret, medie suspendit elocutionem, non ut religionem praetenderet, sed ut crimen.

Inutile de multiplier les exemples. Un même esprit anime de toute évidence les scholiastes grecs et les commentateurs latins. La liberté des uns et des autres est une liberté contrôlée, se déployant dans les limites bien comprises de l'exégèse philologique, c'est-à-dire d'une intelligence soumise pour ainsi dire à une forme de pertinence empathique, où ce qui importe, c'est la conformité de l'interprétation à l'intention, par abdication de la sub-

12 Servii Grammatici qui feruntur in Vergilii Aeneidos libros I-III commentarii, rec. G. Thilo, Leipzig, B. G. Teubner, 1878.

13 Ciceronis orationum scholiastae Asconius scholia Bobiensia. Scholia Pseudasconii Sangallensia. Scholia Cluniacensia et recentiora Ambrosiana Acuaticana. Scholia Lugdunensia sive Gronoviana et eorum excerpta Lugdunensia, rec. Th. Stangl, II, Commentarios continens, Wien, F. Tempsky, Leipzig, G. Freytag, 1912.

EMERITA. Revista de Lingüística y Filología Clásica (EM) — LXXIII 1, 2005 ISSN 0013-6662 
jectivité devant le postulat souverain de l'objectivité érudite. Dans les passages suivants, le commentateur suspend son jugement, comme cela arrive assez fréquemment au reste, et se contente d'éclairer les ressorts de la polysémie qui lui apparaît:

Énéide I 82. AGMINE uel impetu uel multitudine. agmen enim polysemus sermo est. nam impetum significat, ut illi agmine certo Laocoonta petunt; multitudinem, ut uocat agmina saeua sororum. $\| 122$. VICIT HIEMS 'hiems' duas res significat: aut tempus aut uim uenti ... $\| 464$. INANI epitheton est picturae, aut quia caret corporum quae imitatur plenitudine, aut quia nullius est utilitatis...

Énéide IV 5. NEC PLACIDAM MEMBRIS DAT CVRA QVIETEM aut penitus quiete caruit, ut 'placida' epitheton sit quietis: aut habuit quidem quietem, sed non placidam, id est turbatam somniis: unde et ipsa paulo post 'quae me suspensam insomnia terrent'.

Plus rarement, le commentateur prend la liberté d'émettre un jugement, comme dans le passage suivant de Virgile, où apparaît le verbe convertere:

Énéide I 409. VERAS AVDIRE ET REDDERE VOCES? sunt multae reciprocae elocutiones, ut hoc loco; sunt multae unius partis utrique sufficientes, ut 'tenemur amicitiis', ridiculum enim est si addas 'mutuis', cum amicitiae utrumque significent, sicut Fronto testatur. item sunt elocutiones, quarum una pars plena est: quae si conuertantur, habent aliquid superfluum.

Si le lexique constitue le domaine le plus largement, et en quelque sorte le plus aisément, représenté dans le commentaire philologique, les autres catégories linguistiques ne sont pas négligées pour autant : syntaxe, morphologie, morphosyntaxe, morpholexique, dialectologie, stylistique se trouvent pris tour à tour ou simultanément comme objets d'analyse par les commentateurs, plus volontiers curieux toutefois des unités minimales que des structures syntagmatiques ou phrastiques supérieures. La métalangue exégétique, comme la langue tout court, prend pour objets des entités, linguistiques ou extralinguistiques, procédant du libre choix des locuteurs et des scripteurs. La métalangue des scholies illustre de toute évidence une forme très particulière d'arbitraire herméneutique. Le choix porté sur tel ou tel mot ou passage à commenter est déjà connotatif. Il procède d'une sorte de limitation cognitive, qui caractérise en propre la logique schédographique. Le texte second que les scholies dessinent autour du texte premier n'en est ni le reflet mimétique ni la paraphrase exhaustive et continue. C'est un paratexte, un à-côté partial et incomplet, une glose sélective et discontinue et, du point de vue des éditions modernes, une concaténation à choix multiples. Avant toute autre chose, le texte des scholies dénote la rencontre entre un regard ou un esprit et une œuvre, un stade postérieur et un stade antérieur dans la chrono- 
logie objective de l'histoire de la tradition. Dénotant cette rencontre, les scholies se trouvent placées épistémiquement du côté de la connotation, autrement dit de l'ajout, du surcroît, du complément, du com-mentaire tout simplement. Leur nature fondamentale et première est parasitaire, dépourvue en elle-même d'une réalité véritable et autonome. Le texte des scholies serait-il par conséquent un non-texte, que caractérise et affaiblit à la fois la conciliation impossible entre exigence dénotative et orientation connotative?

Les corpus des scholies n'existent que rétrospectivement, du point de vue des modernes. Originellement, chaque annotation est autonome et isolée, se greffant marginalement sur le texte d'origine, n'aspirant qu'à éclairer le lecteur occasionnel et ignorante du sort que la postérité allait lui réserver. La scholie dénote le texte-source en attestant son existence et la réalité de sa lecture; la scholie connote le texte-source en l'assortissant d'une interprétation requise par les besoins supposés de la compréhension. Chaque philologue moderne est en mesure d'apprécier le mélange d'intelligence et d'étrangeté qui compose la matière des scholies. Pour le dire autrement, sans elles le lecteur moderne serait-il vraiment désorienté; qu'apportent-elles que les lexiques, les traductions et la tradition en général n'eussent de toute façon permis de conserver pour les besoins de l'intelligibilité? Les scholies d'emblée ouvrent les textes anciens à un au-delà, leur assurant continuité et prolongement. Elles constituent l'héritage antique en réservoir de sens, et les langues anciennes en expression originelle du Verbe universel.

Dès les origines, la philologie manifeste l'étendue de ses possibilités en même temps qu'elle trahit les risques de l'hypertrophie exégétique, c'est-àdire de l'explication inutile ou superflue, du commentaire inadéquat ou incongru. Les éditions commentées de la Renaissance et au delà, en perpétuant une tradition jamais mise en cause, font apparaître en plus d'un endroit les mirages voire les pièges d'une pratique philologique devenant à ellemême sa propre fin, donnant libre cours à l'accroissement illimité de l'excroissance. D'où l'idée qu'au-delà des signifiants, des syntagmes, des phrases ou des pensées que les scholies expliquent, glosent ou commentent, c'est l'antiquité tout entière que le commentaire philologique dénote et connote, sans fin et par tous les moyens, jusqu' au vertige de l'éternité que la philologie se propose précisément d'incarner. 